\title{
Central Nervous System Fibrosis Is Associated with Fibrocyte-Like Infiltrates
}

\author{
Amy Aldrich and Tammy Kielian \\ From the Department of Pathology and Microbiology, University \\ of Nebraska Medical Center, Omaha, Nebraska
}

Fibrotic wall formation is essential for limiting pathogen dissemination during brain abscess development. However, little is known about the regulation of fibrotic processes in the central nervous system (CNS). Most CNS injury responses are associated with hypertrophy of resident astrocytes, a process termed reactive gliosis. Studies of fibrosis outside the CNS have identified two bone marrow-derived cell types, fibrocytes and alternatively activated M2 macrophages, as key mediators of fibrosis. The current study used bone marrow chimeras generated from green fluorescent protein transgenic mice to evaluate the appearance of these cell types and whether bone marrowderived cells were capable of acquiring fibrotic characteristics during brain abscess development. Immunofluorescence staining revealed partial overlap between green fluorescent protein, $\alpha$-smooth muscle actin, and procollagen, suggesting that a population of cells forming the brain abscess capsule originate from a bone marrow precursor. In addition, the influx of fibrocyte-like cells into brain abscesses immediately preceded the onset of fibrotic encapsulation. Fibrotic wall formation was also associated with increased numbers of alternatively activated M2 microglia and macrophages. To our knowledge, this is the first study demonstrating that bone marrow-derived infiltrates are capable of expressing fibrotic molecules during CNS inflammation. (Am J Pathol 2011, 179:2952-2962; DOI: 10.1016/j.ajpath.2011.08.036)

Staphylococcus aureus is a common etiological agent of brain abscesses in humans, estimated to account for 1 in 10,000 hospital admissions annually of an infectious disease nature. ${ }^{1}$ Our laboratory has developed a mouse model of $S$. aureus-induced brain abscess that has facilitated identification of the pathological changes that occur throughout the course of infection. ${ }^{2}$ In this model, brain abscesses initiate with an early cerebritis response, which spans from 1 to 3 days after bacterial infection. A late cerebritis stage ensues and is immediately followed by capsule formation from day 7 onward. ${ }^{3}$ The process of brain abscess encapsulation involves the formation of a fibrotic wall, which functions to sequester the lesion and protect surrounding parenchyma from additional destruction. Previous studies have demonstrated that myofibroblasts, typified by their hallmark expression of $\alpha$-smooth muscle actin ( $\alpha$-SMA), comprise the fibrotic abscess capsule along with extensive type I collagen and fibronectin deposition. ${ }^{4}$ Importantly, abscess encapsulation ensues in response to diverse central nervous system (CNS) pathogens, such as bacteria, parasites, and fungi, suggesting the induction of a conserved core fibrotic response. ${ }^{1}$

Myofibroblasts are a principle cell type responsible for extracellular matrix (ECM) deposition and wound contraction. ${ }^{5}$ Myofibroblasts can arise from multiple cell types including: i) differentiation from fibrocytes, a bone marrow-derived precursor present in the systemic circulation; ii) transition from epithelial cells; or iii) transformation from resident pericytes and/or smooth muscle cells associated with the vasculature. ${ }^{6}$ As previously mentioned, studies have established the presence of $\alpha$-SMA ${ }^{+}$ myofibroblasts within the brain abscess wall that express type I collagen and fibronectin. ${ }^{4,7}$ Despite this information, the cellular origin of these abscess-associated myofibroblasts remains elusive since the CNS parenchyma is devoid of any resident fibroblast population. Meningeal fibroblasts are not a likely candidate because there is typically no direct path of communication between the meninges and deep brain parenchyma where many brain abscesses arise. In addition, it is unlikely that myofibroblasts are derived from an epithelial precursor because the brain is not associated with an epithelial layer. This

Supported by the $\mathrm{NIH} /$ National Institute of Neurological Disorders and Stroke (NINDS) 2 R01 NS055385 (T.K.).

Accepted for publication August 18, 2011.

Supplemental material for this article can be found at http://ajp. amjpathol.org or at doi: 10.1016/j.ajpath.2011.08.036.

Address reprint requests to Tammy Kielian, Ph.D., University of Nebraska Medical Center, Department of Pathology and Microbiology, 985900 Nebraska Medical Center, Omaha, NE 68198-5900. E-mail: tkielian@unmc.edu. 
leaves bone marrow-derived fibrocytes and/or cells that transdifferentiate from existing vasculature as likely candidates for myofibroblast origin in the context of CNS fibrosis. Of interest to this study is the fibrocyte, which is present in the peripheral circulation and is defined by its expression of CD45, CXCR4, and type I collagen. ${ }^{6,8} \mathrm{Fi}$ brocytes are recruited to sites of tissue injury, whereupon they secrete cytokines that impact ECM and matrix metalloproteinase production to facilitate wound healing. ${ }^{9}$ Brain abscesses are typified by extensive necrosis coupled with a sizable peripheral immune cell infiltrate; therefore, it appeared plausible that fibrocytes may be recruited and driven to differentiate into myofibroblasts to facilitate abscess encapsulation during later stages of infection.

Microglia and macrophages represent two key cell types involved in CNS immune responses to microbial infection through their robust production of proinflammatory mediators, phagocytic activity, and antigen-presenting ability. ${ }^{10} \mathrm{~A}$ pivotal role for microglia in initial bacterial recognition events in the CNS was demonstrated by a recent study from our group in which MyD88, an essential adaptor for Toll-like receptor and IL-1R/L-18 signaling, was essential within the CNS compartment for the genesis of protective immunity during early brain abscess development. ${ }^{11}$ In addition to orchestrating antimicrobial responses, macrophages and microglia can also facilitate inflammation resolution and wound healing processes. In particular, studies have demonstrated that macrophages, and more recently microglia, can be programmed as classically activated $\mathrm{M} 1$ or, alternatively activated M2 cells, depending on the local cytokine milieu. ${ }^{12}$ For example, proinflammatory cytokines such as TNF- $\alpha$ and IFN- $\gamma$ induce classically activated M1 macrophages/microglia that exert potent inflammatory and microbicidal activities. In contrast, IL-4, -5 , and -13 lead to alternative $\mathrm{M} 2$ activation that is associated with inflammation resolution and fibrosis. ${ }^{13}$ Both $\mathrm{M} 1$ and M2 macrophages/microglia compete for the same substrate (ie, L-arginine) to partially regulate their effector functions. For example, inducible nitric oxide synthase (iNOS) expression by $\mathrm{M} 1$ macrophages/microglia utilizes L-arginine for generating NO, which is directly involved in pathogen killing and inflammation. Conversely, M2 macrophages/ microglia express high levels of arginase-1, which metabolizes L-arginine to form proline, the principle substrate for collagen formation. ${ }^{14}$ Because collagen represents a major component of the fibrotic brain abscess capsule, the presence of alternatively activated M2 microglia/macrophages appeared likely but has not yet been demonstrated.

Here, we examined the appearance and infiltration kinetics of fibrocytes, as well as M2 macrophages and microglia, into evolving brain abscesses and how these indices coincided with the time course of fibrotic wall formation. Because brain abscesses are typified by the presence of both activated CNS resident cells, as well as peripheral immune cell populations, we used green fluorescent protein (GFP) bone marrow chimera mice to discriminate between these two populations. GFP chimeras revealed the influx of bone marrow-derived, fibrocyte- like cells $\left(\mathrm{CD}_{4} 5^{+}, \mathrm{CXCR} 4^{+}\right.$, collagen $^{+}, \mathrm{F} 4 / 80^{+}$, and $\mathrm{CD}_{11 \mathrm{~b}^{+}}$) into the infected CNS, which mirrored the kinetics of abscess encapsulation. Similarly, M2 macrophages and microglia were localized along the abscess wall, and markers for these cells were also found to increase in accordance with fibrotic wall formation. Importantly, the myofibroblast marker $\alpha$-SMA, as well as procollagen, demonstrated overlap with infiltrating GFP ${ }^{+}$ cells, indicating that a subset of myofibroblasts originated from a bone marrow precursor. Understanding the pathway(s) regulating CNS fibrosis may allow for future manipulation to contain areas of damage following CNS infection, trauma, or stroke and minimize bystander destruction of surrounding normal parenchyma.

\section{Materials and Methods}

\section{Generation of Experimental Brain Abscesses}

A methicillin-resistant S. aureus (MRSA) USA300 strain, which was recovered from an otherwise healthy individual that died from a brain abscess, was used for these studies. ${ }^{15}$ Bacteria grown to log-phase were encapsulated in agarose beads, and brain abscesses were induced in C57BL/6 mice (Charles River Laboratories, Frederick, MD), using a stereotaxic injection approach as previously described, ${ }^{2}$ whereas control animals received sterile agarose beads. Briefly, mice were anesthetized with $2.5 \%$ avertin i.p. and a $1-\mathrm{cm}$ longitudinal incision was made in the scalp. A rodent stereotaxic apparatus equipped with a Cunningham mouse adaptor (Stoelting, Kiel, WI) was used to implant S. aureus-encapsulated beads into the striatum using the following coordinates relative to bregma: $+1.0 \mathrm{~mm}$ rostral, $+2.0 \mathrm{~mm}$ lateral, and $-3.0 \mathrm{~mm}$ deep from the brain surface. A burr hole was made and a $10-\mu \mathrm{L}$ Hamilton syringe fitted with a 26-gauge needle was used to slowly deliver $2 \mu \mathrm{L}$ of $S$. aureus-laden beads $\left(8 \times 10^{3}\right.$ to $1 \times 10^{4}$ colony forming units) into the brain parenchyma. The incision was closed using Vetbond (3M, St. Paul, MN), and mice were regularly monitored throughout the course of infection. The animal use protocol, approved by the University of $\mathrm{Ne}$ braska Medical Center Animal Care and Use Committee, is in accord with the National Institutes of Health guidelines for the use of rodents.

\section{Generation of Radiation Bone Marrow Chimera Mice}

Bone marrow chimeras were generated using C57BL/6 mice as recipients and C57BL/6-Tg(CAG-EGFP) mice (The Jackson Laboratory; Bar Harbor, ME) as donors as previously described with minor modifications. ${ }^{11}$ This paradigm allowed for the direct visualization of infiltrating $\mathrm{GFP}^{+}$bone marrow-derived cells and an assessment of fibrotic molecule expression by signal colocalization following immunofluorescent staining. In addition, B6/SJL mice that are congenic for the CD45 allele (CD45.1) on a C57BL/6 background were purchased from The Jackson Laboratory and used to generate bone marrow chimeras 
with C57BL/6 mice (CD45.2) for some fluorescence activated cell-sorting (FACS) studies. Briefly, GFP ${ }^{+}$or B6/ SJL donor mice were euthanized, and spleens and long bones harvested. Subsequently, bones were flushed using sterile PBS and spleens passed through a $70-\mu \mathrm{m}$ filter and triturated to obtain a single-cell suspension. C57BL/6 recipient mice were irradiated (1200 rad) using a J.L. Shepard Mark I 68A cesium irradiator and immediately received retro-orbital injections of $2 \times 10^{7}$ bone marrow cells supplemented with $10^{7}$ splenocytes, the latter serving as an immediate source of immune cells. Engraftment occurred over a 6- to 8-week period, and chimeric animals were maintained on antibiotic-supplemented water ( $1 \mathrm{~g} / \mathrm{L}$ neomycin and $125 \mathrm{mg} / \mathrm{L}$ polymyxin) for 2 weeks to provide protection during transient immunocompromise. At 6 to 8 weeks after transplant, chimeric mice were bled retro-orbitally, and leukocytes were evaluated by flow cytometric analysis to verify chimerism by determining the percentage of $\mathrm{GFP}^{+}$or $\mathrm{CD} 45.1^{+}$cells in the systemic circulation. Only animals that displayed chimerism of greater than $90 \%$ were evaluated at approximately 10 to 12 weeks following bone marrow transfer, a period that we have established to be sufficient for establishing chimerism. ${ }^{11}$ Importantly, in our studies, parenchymal microglia were not repopulated with $\mathrm{GFP}^{+}$cells as has been described by others, ${ }^{16}$ although $\mathrm{GFP}^{+}$cells were associated with blood vessels, representing perivascular macrophages (see Supplemental Figure S1 at http:// ajp.amjpathol.org).

\section{Bacterial Quantification in Brain Abscesses}

Enumeration of viable abscess-associated bacteria was determined by plating serial 10-fold dilutions of brain homogenates on $5 \%$ sheep blood agar plates. Titers are expressed as colony-forming units per gram wet tissue weight.

\section{Immunofluorescence Staining and Confocal Microscopy}

GFP bone marrow chimera mice were euthanized at days 7 and 14 following infection to collect brain abscess tissues for immunofluorescence staining. Briefly, a catheter was inserted into the left cardiac ventricle and the vasculature cleared by a 2-minute perfusion with PBS followed by $4 \%$ paraformaldehyde for 4 minutes (flow rate $=10 \mathrm{~mL} / \mathrm{min}$ ), whereupon brains were extracted and postfixed in $4 \%$ paraformaldehyde for 1 hour at $4^{\circ} \mathrm{C}$. Next, brains were cryoprotected using a sucrose gradient and stored in 30\% sucrose for 24 hours, whereupon tissues were imbedded in Optimal Cutting Temperature medium and frozen on dry ice for subsequent cryostat sectioning as described below.

To prepare frozen sections for staining, $10-\mu \mathrm{m}$-thick sections of brain abscess tissues were cut using a MICROM HM550 cryostat (Reichert-Jung; Leica Microsystems, Wetzlar, Germany). Tissue sections were fixed with ice-cold methanol and stained with antibodies specific for arginase-1, procollagen (both from Santa Cruz
Biotechnology, Santa Cruz, CA), iNOS (Abcam, Cambridge, MA), $\alpha$-SMA (Epitomics, Burlingame, CA), type I collagen (Millipore, Billerica, MA), type II collagen (Novus Biologicals, Littleton, CO), laminin (Sigma, St. Louis, MO), fibronectin (US Biological, Swampscott, MA), and Iba-1 (Biocare Medical, Concord, NH). For arginase-1, procollagen, $\alpha$-SMA type I and type II collagen, laminin, fibronectin, and Iba-1, a biotinylated secondary antibody was used followed by a streptavidin-594 conjugate (Jackson ImmunoResearch Laboratories, West Grove, PA). For three-color staining, a DyLight 405-conjugated secondary antibody (Jackson ImmunoResearch Laboratories) was used to visualize procollagen. Confocal images were acquired using a Zeiss 510 META laser scanning microscope (Carl Zeiss, Oberkochen, Germany) with tissues stained with secondary antibodies only as a negative control.

\section{Flow Cytometry}

Brain abscess tissues were collected and processed for flow cytometry as previously described. ${ }^{11,17}$ Briefly, GFP bone marrow chimera mice were perfused to remove circulating leukocytes from the vasculature, whereupon brain abscess tissues were minced and filtered through a 70- $\mu \mathrm{m}$ nylon mesh cell strainer. The resulting homogenate was subjected to collagenase/DNase I digestion (both from Sigma-Aldrich) for 30 minutes, and after enzyme neutralization, cells were applied to a Percoll gradient (1.03 to $1.088 \mathrm{~g} / \mathrm{mL}$ ). After centrifugation, the myelin layer was aspirated and the cellular interface collected. $\mathrm{GFP}^{+}$cells were sorted using a BD FACSAria, incubated with Fc Block (BD Biosciences, San Jose, CA), and stained with CXCR4-PE, CD45-APC, CD11b-Percp Cy5.5, and F4/80-Alexa 488 (all from BD Biosciences). Type I collagen (Millipore) was visualized by intracellular staining using anti-rabbit PerCP-Cy 5.5 (Santa Cruz Biotechnology). $\mathrm{GFP}^{+}, \mathrm{CD} 5^{+}, \mathrm{CXCR} 4^{+}$, and collagen- ${ }^{+}$ cells were defined as fibrocyte-like cells because of their concomitant expression of the macrophage marker F4/80 (see Supplemental Figure S2 at http://ajp. amjpathol.org). ${ }^{6,18}$

To determine the percentages of $\mathrm{CD}_{206}{ }^{+} \mathrm{M} 2$ macrophages and microglia associated with brain abscesses, cells were collected from lesions as described above and stained with CD45-APC and CD11b-Percp Cy5.5 (both from BD). Macrophages and microglia were sorted using a BD FACSAria on the basis of differential CD45 expression (CD45 ${ }^{\text {hi }}$ and CD45 intermediate-low, respectively) and subsequently stained with CD206-FITC (Biolegend, San Diego, CA) to identify M2 alternatively activated cells.

\section{qPCR}

Brain tissues were collected and RNA isolated using TriZol (Invitrogen, Carlsbad, CA) and processed into cDNA using the iScript cDNA synthesis kit (BioRad; Hercules, $\mathrm{CA}$ ) according to the manufacturer's instructions. $\mathrm{ABI}$ Assays on Demand primer/probe sets (Applied Biosystems, Foster City, CA) were used for quantitative real-time PCR (qPCR) to evaluate iNOS, arginase-1, chitinase-3 


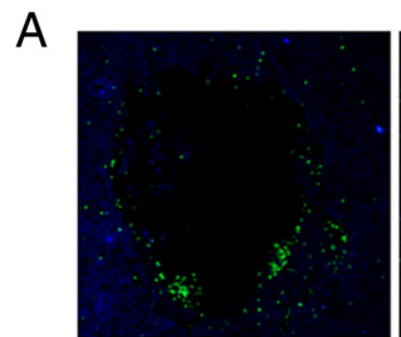

Day 2

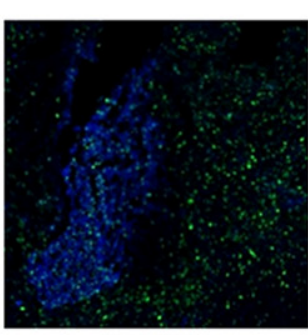

Day 5

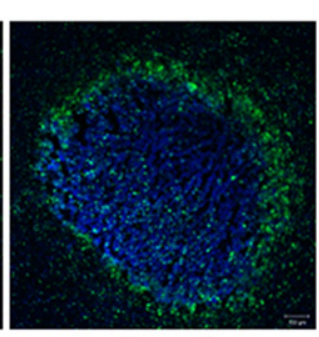

Day 7
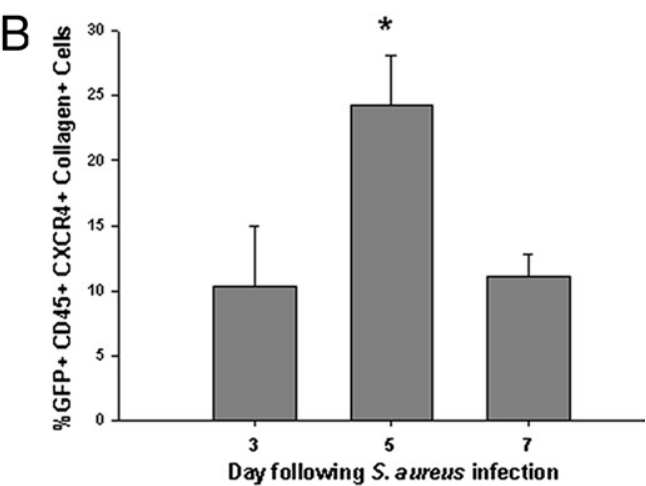

Figure 1. Bone marrow derived fibrocyte-like cells infiltrate the brain during abscess formation. Brain abscesses were induced in GFP bone marrow chimera mice to track the appearance and localization of infiltrating bone marrow-derived cells during infection. A: Localization patterns of infiltrating cells differ depending on the stage of infection. B: The kinetics of fibrocyte $\left(\mathrm{GFP}^{+}, \mathrm{CD} 45^{+}, \mathrm{CXCR}^{+}\right.$, collagen $\left.{ }^{+}\right)$infiltration into brain abscesses was quantified by FACS with results combined from a total of four independent experiments (mean \pm SEM). ${ }^{*} P<0.05$ versus fibrocyte-like infiltrates at day 3 .

(YM-1), triggering receptor expressed on myeloid cells 1 (TREM-1), TREM-2, transforming growth factor- $\beta$ (TGF$\beta$ ), signal transducer and activator of transcription 6 (STAT6), hypoxia-inducible factor-1 $\alpha$ (HIF-1 $\alpha)$, type I collagen, fibronectin, and matrix metalloproteinase- 9 (MMP-9) levels. Relative gene expression levels were calculated after normalizing cycle thresholds against the housekeeping gene GAPDH and are presented as the fold-induction $\left(2^{-\Delta \Delta C t}\right)$ value relative to an animal injected with sterile agarose beads as a reference standard.

\section{Western Blot Analysis}

To evaluate the expression of select markers associated with M1 versus M2 macrophages/microglia in brain abscesses, Western blots were performed as previously described. ${ }^{19}$ Blots were probed with antibodies specific for arginase-1 (Santa Cruz Biotechnology) or YM-1 (R\&D Systems, Minneapolis, MN) and were stripped and reprobed for $\beta$-actin (Sigma) to serve as a loading control. An Alpha Innotech HD2 gel documentation system (San Leandro, CA) was used to quantify chemiluminescent intensity and relative protein expression, which was calculated by normalizing band intensity values of the protein of interest by $\beta$-actin of the same sample.

\section{Statistical Analysis}

Differences between experimental groups were determined using a one-way analysis of variance (analysis of variance) followed by Tukey's Multiple Comparisons Test (GraphPad Prism; GraphPad Software, La Jolla, CA). For all analyses, a $P$ value of less than 0.05 was considered statistically significant.

\section{Results}

\section{Bone Marrow-Derived Fibrocyte-Like Cells Infiltrate Brain Abscesses}

To facilitate the visualization and distribution of bone marrow-derived cells infiltrating brain abscesses, GFP bone marrow chimera mice were generated. In our studies, significant repopulation of parenchymal microglia was not observed, although $\mathrm{GFP}^{+}$perivascular macrophages were evident (see Supplemental Figure S1 at http://ajp. amjpathol.org). Infiltrating $\mathrm{GFP}^{+}$cells were detected at day 2 after infection and progressively increased at day 5 when they were distributed diffusely throughout lesions (Figure 1A). In contrast, GFP ${ }^{+}$infiltrates were highly organized along the abscess periphery by day 7 after infection, which coincides with the location of the developing fibrotic wall. The distinct colocalization patterns of infiltrating bone marrow-derived cells with the fibrotic capsule suggest that they may influence abscess encapsulation.

Previous studies have established that the brain abscess wall is composed of myofibroblasts as evident by their hallmark expression of $\alpha$-SMA, as well as numerous ECM components including type I collagen and fibronectin. ${ }^{4,7}$ Since the CNS parenchyma is devoid of a resident fibroblast population, we examined the possibility that myofibroblasts may, at least in part, originate from a bone marrow-derived cell type. One attractive candidate was the fibrocyte, a bone marrow-derived cell present in the peripheral circulation and shown to differentiate into fibroblasts/myofibroblasts following invasion into target tissues such as the lung and liver. ${ }^{20,21}$ Fibrocytes express CD45, CXCR4, and collagen, ${ }^{6,18}$ and in addition to these molecules, the use of GFP bone marrow chimeras provided us with an additional discriminative marker based on their bone marrow derivation. GFP $^{+}$cells were isolated from brain abscesses at various intervals after infection by FACS and were defined as fibrocytes based on CD45, CXCR4, and type I collagen staining. Fibrocyte infiltrates were already apparent at day 3 and were maximal at day 5 after infection (Figure 1B). However, these cells were still detected at day 7 suggesting continued recruitment at a time when fibrotic wall formation has initiated. Further analysis of infiltrating fibrocytes revealed CD11b and F4/80 coexpression, suggesting that they may also arise from a macrophage-like precursor (see Supplemental Figure 2 at http://ajp.amjpathol.org). To further address the issue of fibrocyte origin, we performed staining for CD13 and CD34, two stem cell markers that 
have also been reported to be expressed by fibrocytes. ${ }^{6}$ The $\mathrm{CD}_{4} 5^{+}, \mathrm{CXCR}^{+}$, collagen ${ }^{+}$population was also $\mathrm{CD}_{13^{\mathrm{lo}}}$ and $\mathrm{CD} 34^{\mathrm{lo}}$ (data not shown), further suggesting that these cells represent fibrocytes since these markers are typically associated with stem cells and fibrocytes, in particular. Because of their rather promiscuous surface marker expression, these cells are referred to as "fibrocyte-like" throughout the remainder of the paper. These findings suggest that fibrocyte-like cells may serve as a precursor to $\alpha-\mathrm{SMA}^{+}$myofibroblasts during CNS infection.

\section{Bone Marrow-Derived Cells Express Fibrosis- Associated Molecules on Entry into the Infected CNS}

To determine whether bone marrow-derived cells were capable of acquiring myofibroblast characteristics, immunofluorescence staining was performed on brain abscess tissues from GFP bone marrow chimera mice. $\alpha$-SMA, procollagen, and type II collagen demonstrated overlap with $\mathrm{GFP}^{+}$cells, suggesting that bone marrowderived cells are capable of acquiring characteristics typical of myofibroblasts (Figure 2A). However, it is important to note that not all $\mathrm{GFP}^{+}$cells expressed these molecules, indicating that additional cell type(s) are likely contributing to fibrotic wall formation. Interestingly, procollagen staining was also detected in a subpopulation of $\mathrm{GFP}^{+}$and $\mathrm{lba}-1^{+}$macrophages (see Supplemental Figure 3 at http://ajp.amjpathol.org), which is in agreement with the overlap of macrophage markers with fibrocytelike cells described above. As expected, type I collagen, laminin, and fibronectin staining was prominent at day 14 following infection (Figure 2B). However, no overlap of these molecules with $\mathrm{GFP}^{+}$cells was observed, which was not unexpected since all are secreted molecules. To our knowledge, this is the first demonstration of bone marrow-infiltrating cells expressing fibrotic molecules during CNS infection.

\section{CNS Bacterial Infection Is Typified by Both M1 and M2 Macrophages and Microglia}

Little information is currently available regarding the cellular and molecular events responsible for eliciting fibrosis in the CNS. This is largely due, in part, to the fact that the majority of CNS injury responses are typified by reactive gliosis and glial scar formation. ${ }^{22}$ Since brain abscesses caused by multiple pathogens, including bacteria, fungi, or parasites, are sequestered by a fibrotic capsule, this suggests the involvement of a core fibrotic program. In terms of cellular responses, M2 macrophages and microglia have been shown to promote wound healing/fibrosis that typically ensues during latestage inflammation when the expression of proinflammatory mediators begins to decline. ${ }^{23}$ However, the expression of M2-related markers or M2 macrophages/microglia in the context of CNS infection-associated fibrosis has not yet been examined. Therefore, we used our experimental brain abscess model to address these issues since for-
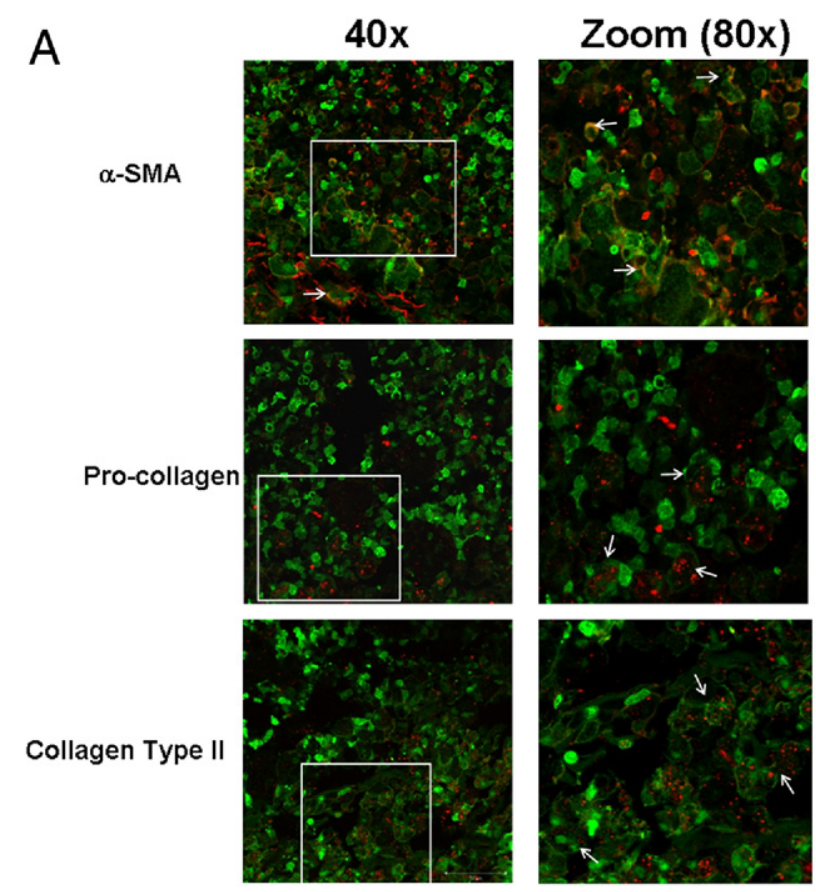

B

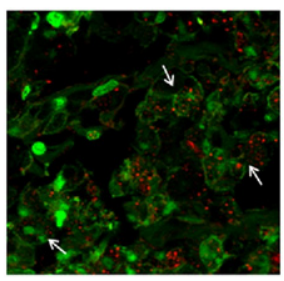

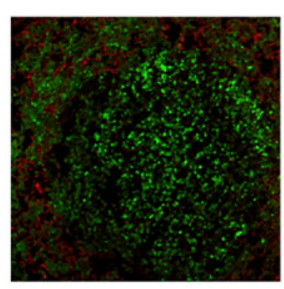

Collagen Type I

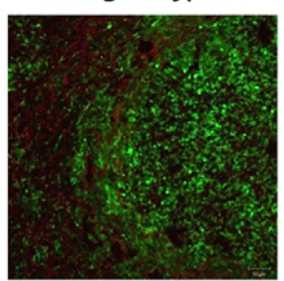

Fibronectin

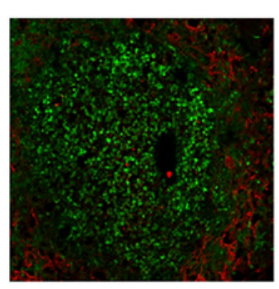

Laminin

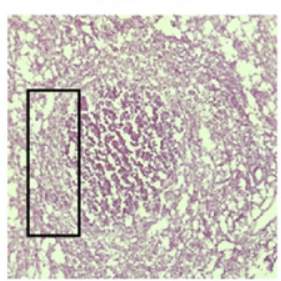

H\&E
Figure 2. Bone marrow-derived cells express fibrosis-associated molecules on entry into the infected CNS. Brain abscesses were induced in GFP bone marrow chimera mice and tissues collected at day 14 following infection for immunofluorescence staining and confocal microscopy. A: Ten-micrometerthick sections were stained for $\alpha$-SMA, procollagen, and type II collagen with staining localized along the brain abscess wall. Zoomed images $(\times 80) \mathrm{dem}-$ onstrated overlap between $\alpha$-SMA, procollagen, and type II collagen (red) with a subset of $\mathrm{GFP}^{+}$cells (green; arrows). B: Significant staining for type I collagen, laminin, and fibronectin (red) was also associated with the brain abscess capsule, and a H\&E image depicting the region where high-power magnifications of staining were determined is delineated by a rectangle. Results are representative of tissues collected from six individual animals.

mation of the fibrotic capsule proceeds with a predictable time course.

Quantitation of molecules associated with M1 vs. M2 macrophages/microglia in brain abscesses by GPCR demonstrated similar expression patterns for the M1-associated markers iNOS and TREM-1 (Figure 3, B and C, respectively), as well as several $\mathrm{M} 2$ molecules including arginase-1, YM-1, and TREM-2 (Figure 3, D-F, respectively). In general, both M1 and M2 marker expression 

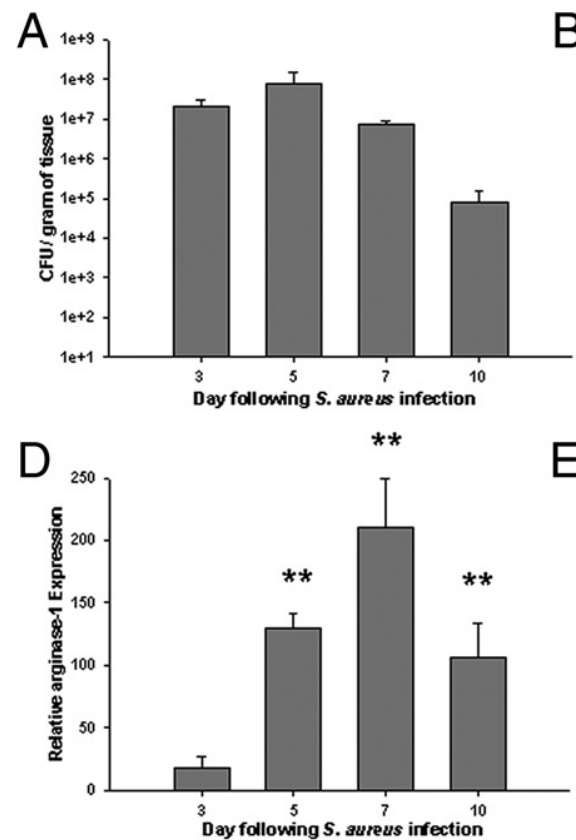
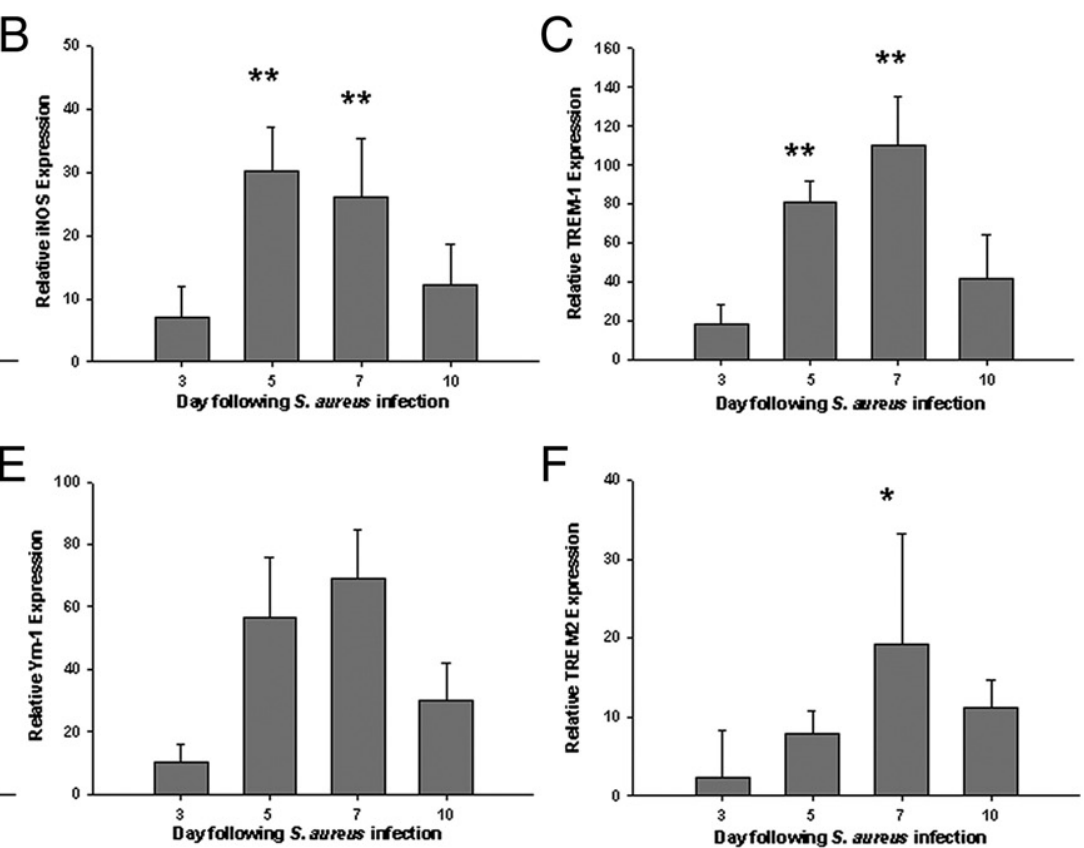

Figure 3. Expression kinetics of M1 and M2 activation markers during brain abscess formation. Brain abscesses were induced in $\mathrm{C} 57 \mathrm{BL} / 6 \mathrm{mice}$ and evaluated at the indicated time points after infection for quantitation of bacterial burdens (A), or markers associated with classically activated M1 (B and C) or alternatively activated M2 (D-F) macrophages/microglia. Gene expression levels in mice harboring brain abscesses were calculated after normalizing signals against GAPDH and are presented as the fold-change relative to sham animals injected with sterile agarose beads as a control. Results represent the mean \pm SD of six to eight individual mice per time point. ${ }^{*} P<0.05,{ }^{* *} P<0.01$ versus day 3 .

peaked at day 7 following infection, the interval in which fibrotic encapsulation of brain abscesses ensues. ${ }^{3}$ These changes also paralleled differences in bacterial burdens (Figure 3A).

To evaluate whether alterations in select M1/M2-associated molecules were also observed at the protein level, Western blot analyses were performed. The relative protein expression of arginase-1, YM-1, and iNOS (Figure 4 and data not shown) mirrored the patterns detected by qPCR (Figure 3), with peak levels observed at day 7 following bacterial exposure (Figure 4). Collectively, these findings demonstrate that both $\mathrm{M} 1$ - and M2-associated markers are coordinately expressed during the course of brain abscess development rather than one phenotype dominating the response. Indeed, recent reports have highlighted similar coexpression patterns of $\mathrm{M} 1$ and M2 profiles during inflammatory processes, sug- gesting that the balance between both may dictate net outcome. ${ }^{12,13}$

To quantitate the extent of alternatively activated M2 macrophages and microglia during the course of abscess formation, FACS analysis was performed for the M2 marker CD206. ${ }^{24}$ For these studies, microglia and macrophages were identified based on differential CD45 expression as previously described. ${ }^{25}$ Interestingly, although both M2 microglia and macrophages were observed throughout the course of brain abscess development, the kinetics of their appearance differed. Namely, maximal CD206 ${ }^{+}$M2 microglia were observed earlier compared to infiltrating CD206 $^{+}$ macrophages (ie, days 5 vs 7 after infection, respectively; Figure 5). In addition, $\mathrm{CD}_{206^{+}}$microglia represented a greater percentage of the population compared to macrophages (Figure 5B), suggesting that M2 microglia may significantly impact early profibrotic responses.

\section{A}
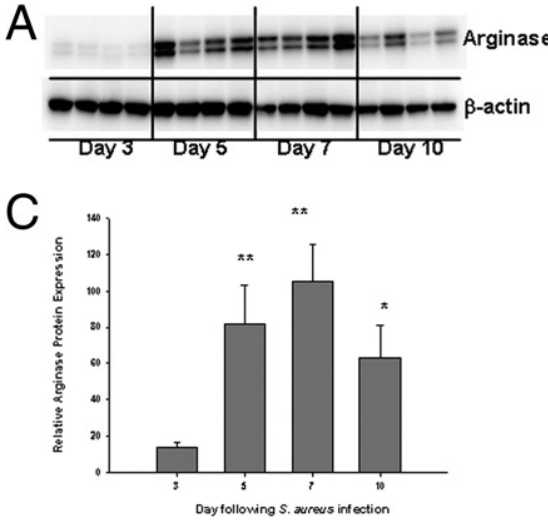

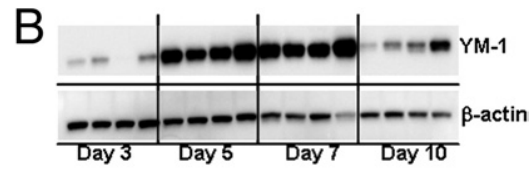

D

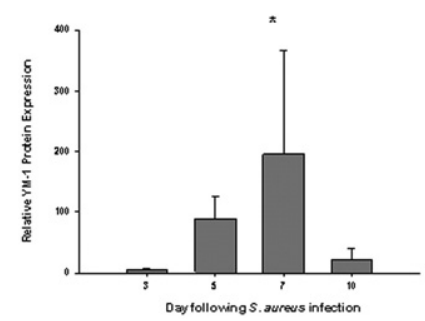

Figure 4. M2 alternative activation marker expression coincides with fibrosis initiation in brain abscesses. Brain abscesses were induced in C57BL/6 mice, whereupon total protein from abscess homogenates was collected at the indicated time points after infection for Western blotting for arginase-1 (A and $\mathbf{C}$ ) and YM-1 (B and $\mathbf{D})$. Blots were stripped and reprobed with an antibody specific for $\beta$-actin to verify uniformity in gel loading. Results are presented from four individual animals per time point and are representative of two independent experiments. ${ }^{*} P<0.05$, ${ }^{* *} P<0.01$ versus day 3 . 

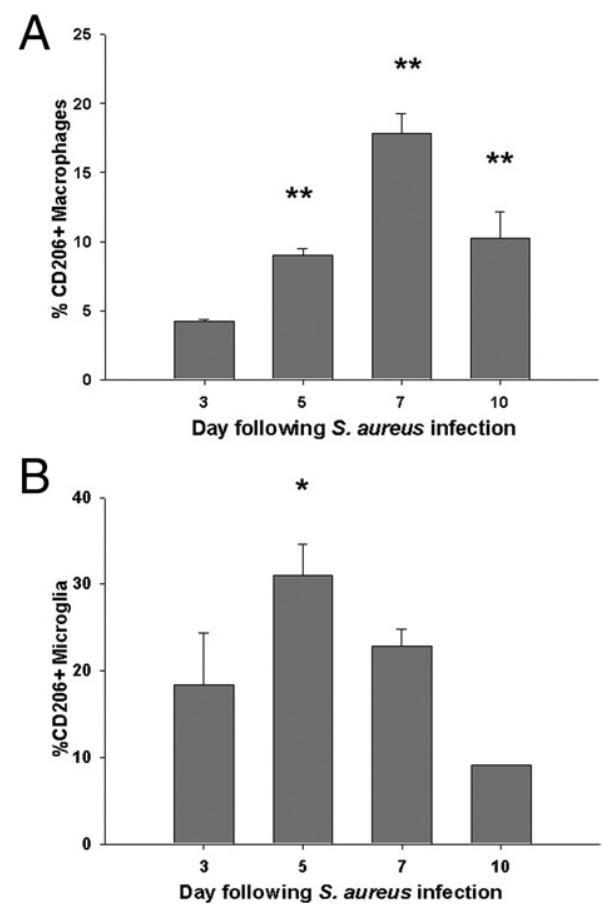

Figure 5. The appearance of M2 macrophages and microglia coincides with the onset of brain abscess fibrotic wall formation. Brain abscesses were induced in $\mathrm{C} 57 \mathrm{BL} / 6$ mice and tissues collected at the indicated time points after infection for quantitation of $\mathrm{M} 2$ macrophages $\left(\mathrm{CD} 11 \mathrm{~b}^{+}, \mathrm{CD} 45^{\text {hi }}\right)$ or microglia (CD11b $\left.{ }^{+}, \mathrm{CD} 45^{\text {lo-intermediate }}\right)$ by FACS using the M2 marker CD206. Results represent the mean \pm SEM from four independent experiments. ${ }^{*} P<$ $0.05,{ }^{* *} P<0.01$ versus day 3 .

\section{M1 and M2 Macrophages/Microglia Localize to Distinct Regions During Brain Abscess Development}

Because both abscess-associated arginase-1 and iNOS expression peaked at day 7 after infection, immunofluorescence staining was performed at this time point to determine the localization patterns of both molecules relative to the developing brain abscess wall. The distribution of arginase-1-positive cells was predominately clustered along the outermost region of the abscess wall at the interface with surrounding uninfected parenchyma (Figure 6A). In addition, many arginase- $1^{+}$cells displayed overlap with $\mathrm{GFP}^{+}$, revealing their bone marrow origin (Figure 6A). In contrast, iNOS ${ }^{+}$cells were distributed more diffusely along the abscess margins (Figure $6 \mathrm{~B})$. The fact that iNOS and arginase- 1 expression appeared to cluster in distinct domains, although some degree of overlap was still visible, suggests the existence of microdomains that contain different inflammatory signals that drive cells to favor the expression of one molecule and effector phenotype.

\section{Characterization of Molecules Associated with CNS Fibrosis}

Although the current findings have demonstrated fibrocyte-like infiltrates as well as M2 microglia and macrophages associated with evolving brain abscesses, the signals contributing to fibrotic wall formation have yet to be defined. The expression of several molecules known to influence various aspects of fibrosis in the periphery was examined to identify candidates that may impact abscess encapsulation. TGF- $\beta$ is a well-characterized fibrotic mediator that can stimulate fibroblast activation and collagen synthesis. ${ }^{26}$ TGF- $\beta$ levels peaked at day 7 following infection, similar to the kinetics of fibronectin expression (Figure 7, A and B, respectively), the latter representing a major constituent of the brain abscess wall. Another component of the fibrotic abscess capsule, type I collagen, was strongly induced throughout the time course examined (Figure 7C). STAT6 plays a key role in the induction of Th2 cytokines such as IL-4 that is important for stimulating fibrotic responses. ${ }^{27}$ STAT6 levels were negligible at day 3 and peaked at day 7 after infection (Figure 7D), which agreed with the kinetics of IL-4 producing Th2 infiltrates into brain abscesses (data not shown). Hypoxia inducible factor- $1 \alpha(\mathrm{HIF}-1 \alpha)$ is a major transcription factor induced in response to hypoxic conditions and can impact fibrosis. ${ }^{28}$ Because of the hypoxic nature of brain abscesses, the expression of this molecule was also investigated. HIF- $1 \alpha$ induction followed a distinct expression pattern with peak levels observed at day 5 after infection followed by a rapid decline (Figure $5 \mathrm{E})$, which precedes the fibrotic and angiogenic responses that occur during brain abscess development. Finally, MMP-9 plays an important role in ECM remodeling and immune cell extravasation across the bloodbrain barrier. ${ }^{29}$ Similar to many of the other profibrotic molecules examined, MMP-9 also showed peak expression at day 7 and declined at day 10 after infection (Figure 5F), a period in which the fibrotic abscess wall has already formed but continues to mature by the continual deposition of fibronectin, type 1 collagen, and laminin. Collectively, these results identify numerous candidates that may impact initiation of the fibrotic abscess wall during CNS infection. The functional importance of each in this process remains to be determined.

\section{Discussion}

Fibrotic wall formation is a pivotal event during brain abscess development, which functions in infection containment and to protect surrounding parenchyma from destruction. However, the exact components of the fibrotic wall and the events that lead to its establishment within the CNS have yet to be completely defined. To the best of our knowledge, this study is the first to implicate the involvement of bone marrow-derived cells in the process of CNS fibrosis. This was demonstrated by the entry of fibrocyte-like cells from the peripheral circulation and the presence of a subpopulation of bone marrow-derived cells that expressed $\alpha$-SMA and procollagen, which were localized to the fibrotic abscess capsule.

The cell type(s) that form the brain abscess wall remained to be identified since the CNS is devoid of any resident fibroblast/myofibroblast population. Although numerous cell types infiltrate the CNS during infection, we focused on fibrocytes, a bone marrow-derived cell 

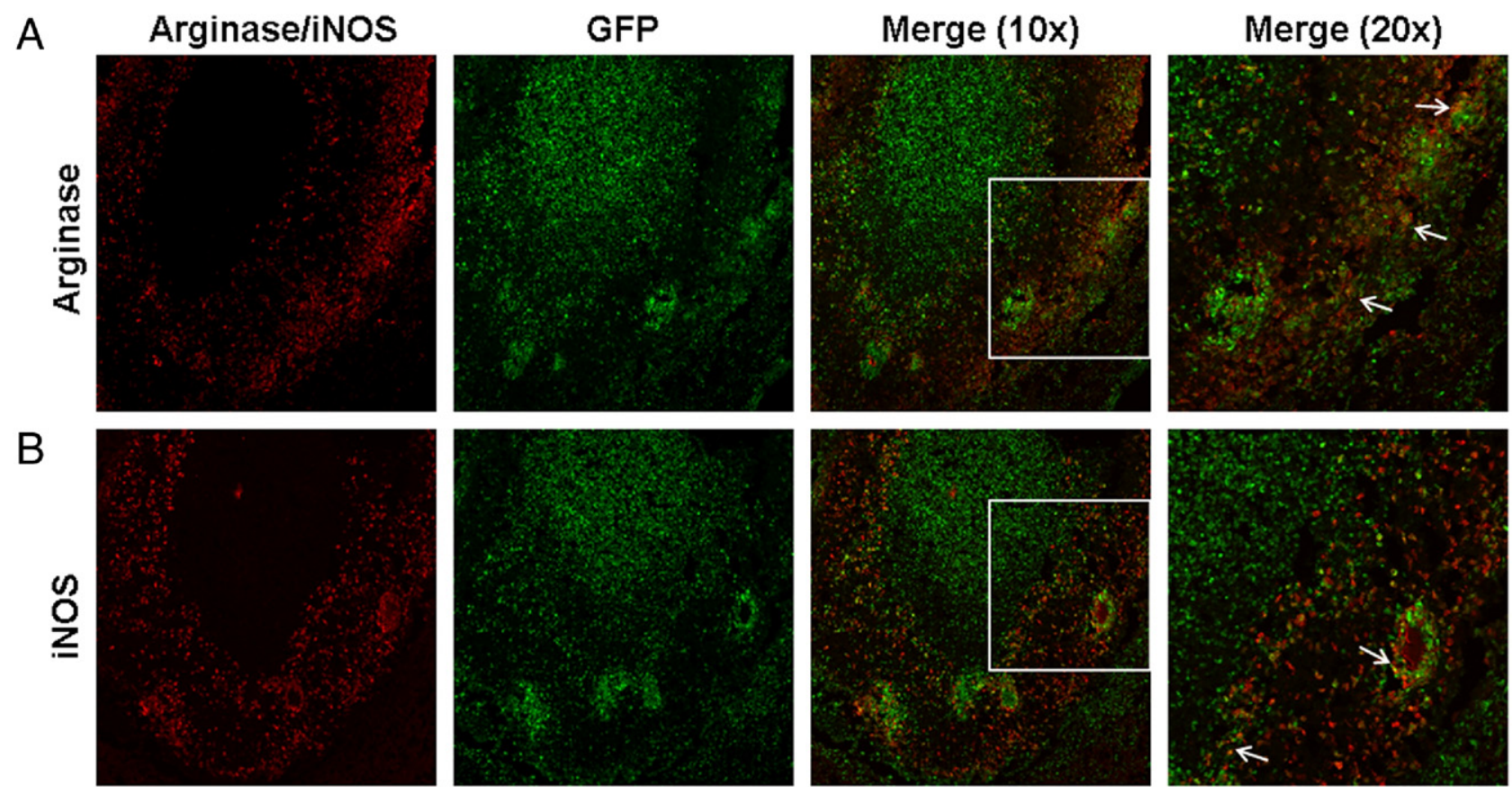

Figure 6. Arginase-1 and iNOS expression is localized to discrete domains within brain abscesses. Brain abscesses were induced in GFP bone marrow chimera mice and tissues collected at day 7 following infection for immunofluorescence staining and confocal microscopy. Sections (10 $\mu \mathrm{m}$ ) were stained for arginase-1 (A) or iNOS (B) with significant overlap of both markers detected in $\mathrm{GFP}^{+}$cells (merge). Arrows highlight areas where overlap between the two stains is observed. Results are representative of tissues collected from six individual animals.

that has been reported to home to areas of tissue injury and differentiate into fibroblasts and myofibroblasts. ${ }^{30,31}$ Based on their characteristic expression of CD45, CXCR4, and type I collagen, fibrocyte-like cells infiltrated brain abscesses as early as day 3 following infection and may represent a precursor to the $\alpha-\mathrm{SMA}^{+}$cells associated with the fibrotic abscess capsule. This possibility was further supported by the finding that a subpopulation
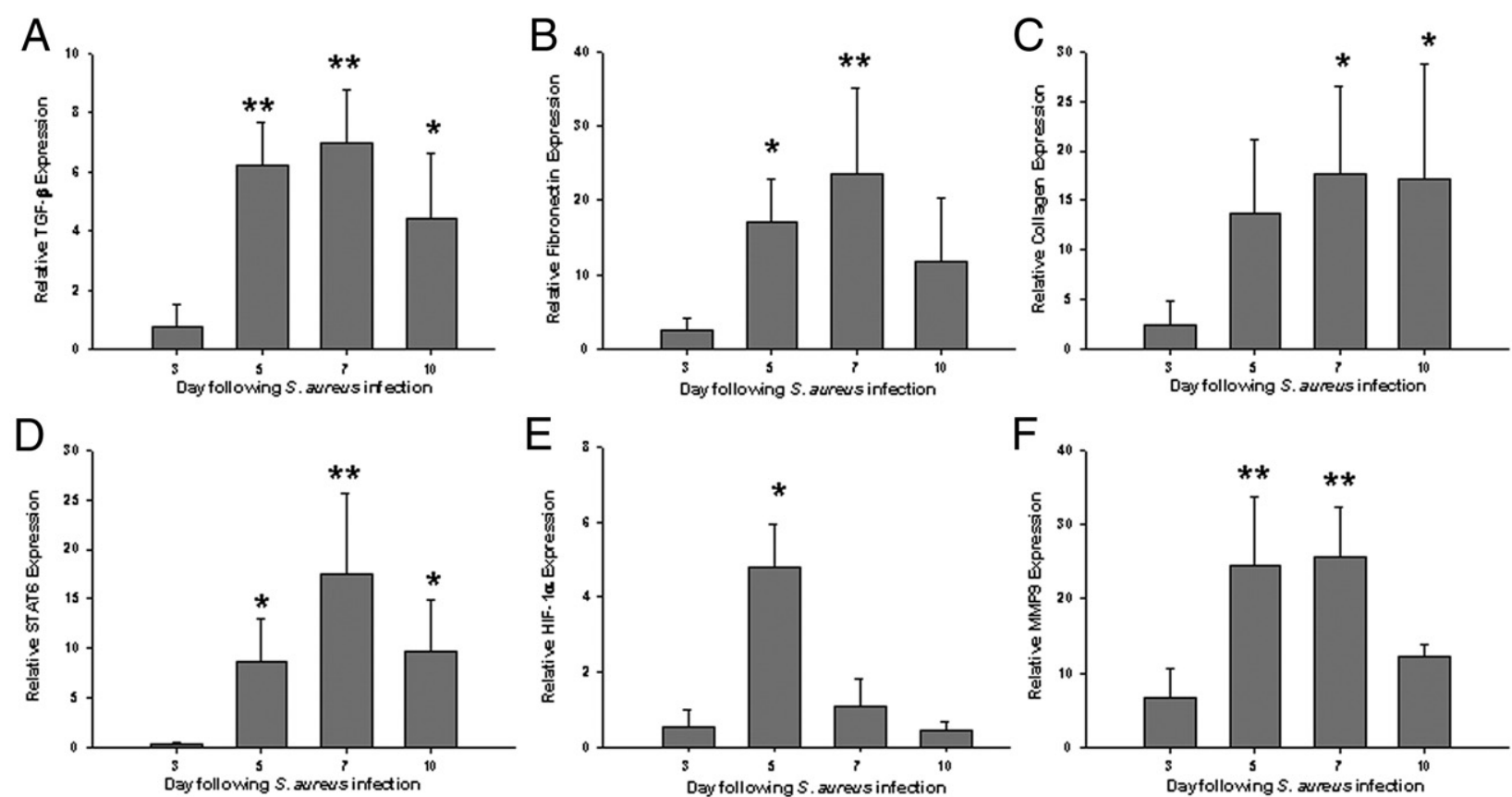

Figure 7. The kinetics of fibrosis regulatory signals coincides with the onset of brain abscess encapsulation. Brain abscesses were induced in $\mathrm{C} 57 \mathrm{BL} / 6$ mice and evaluated at the indicated time points after infection for quantitation of TGF- $\beta$ (A), fibronectin (B), type I collagen (C), STAT6 (D), HIF-1 $\alpha(\mathbf{E})$, and MMP-9 (F) expression by qPCR. Gene expression levels in mice harboring brain abscesses were calculated after normalizing signals against GAPDH and are presented as the fold-change relative to sham animals injected with sterile agarose beads as a control. Results represent the mean \pm SD of six to eight individual mice per time point. ${ }^{*} P<0.05,{ }^{* * *} P<0.01$ versus day 3 . 
of $\alpha$-SMA ${ }^{+}$myofibroblasts bordering the fibrotic wall expressed GFP, demonstrating their bone marrow origin. Interestingly, a recent report suggested that tissue-infiltrating macrophages were capable of differentiating into myofibroblasts. ${ }^{32}$ We did observe nominal overlap of $\mathrm{GFP}^{+}, \mathrm{Iba}-1^{+}$macrophages with procollagen, suggesting that macrophages could acquire myofibroblast-like characteristics. Additional studies are needed to definitively address the frequency of myofibroblast transdifferentiation from macrophages/microglia in the context of CNS fibrosis. Nonetheless, it is clear that alternative cell type(s) also contribute to the population of abscess-associated myofibroblasts, the identity of which remains to be determined. Another possibility is the pericyte, which has been implicated in glial scar formation following spinal cord injury ${ }^{33}$ and reported to differentiate into myofibroblasts. ${ }^{34}$ However, pericyte surface marker expression is highly heterogeneous, making their unequivocal identification difficult. For this reason, many reports examining pericyte function use transgenic mouse models, which will represent an interesting avenue for future studies to determine whether these cells contribute to brain abscess wall formation. It is also important to acknowledge the potential role of astrocytes in abscess containment since studies by others have established that astrocytes can regulate the extent of tissue injury by performing a bordering function. ${ }^{35}$ In addition, reactive astrocytes are known to produce collagen ${ }^{36}$; however, preliminary studies from our laboratory have revealed that astrocytes and $\alpha$-SMA ${ }^{+}$myofibroblasts occupy distinct domains within the abscess and show no degree of overlap (data not shown). Therefore, although astrocytes may provide signals to orchestrate the genesis of the fibrotic abscess capsule, it appears unlikely that they transition into the $\alpha$-SMA ${ }^{+}$cellular elements that comprise the fibrotic wall. Additional studies are currently ongoing in our laboratory to examine the impact of astrocytes on fibrotic processes.

Collagen synthesis by fibroblasts can be induced by several signaling pathways. For example, fibroblasts express IL-4R $\alpha$ and IL-13R $\alpha 1$ and in vitro studies have indicated that both IL-4 and IL-13 are capable of directly stimulating collagen production. ${ }^{37}$ In addition, myofibroblasts express IL-13R $\alpha 2$, which is important for their tissue repair capabilities. ${ }^{38}$ We have demonstrated that both IL-4 and IL-13 producing Th2 cells are present in brain abscesses at later intervals after infection, although the former are more frequent (data not shown). IL-13 can also indirectly regulate collagen production by inducing MMP-9 expression, which leads to the processing of latent TGF- $\beta$ into its active form, making it available to trigger collagen synthesis by fibroblasts/myofibroblasts. ${ }^{39}$ Both TGF- $\beta$ and MMP-9 were coordinately expressed during brain abscess development, suggesting that this mechanism of collagen production may occur during CNS infection, although a direct cause and effect relationship remains to be determined. Another IL-4- and IL-13-driven event that can influence collagen production is alternative M2 macrophage/microglial activation. For example, arginase- 1 activity in alternatively activated M2 macrophages/microglia utilizes L-arginine to produce proline, the main substrate for collagen synthesis. ${ }^{40}$ Conversely, the Th1 cytokine IFN- $\gamma$ antagonizes collagen production by stimulating iNOS, which directly competes with arginase-1 for L-arginine. ${ }^{41}$ In the studies presented here, although arginase-1 and iNOS were coexpressed, the former was more prominent during later stages of infection when profibrotic responses would be expected to be favored. This appears plausible since the time frame for maximal M2 macrophage/microglial numbers coincided with the reduction in abscess-associated $S$. aureus burdens and down-regulation of proinflammatory molecule expression. Indeed, the latter has been implicated as a signal to initiate fibrotic cascades in other wound healing models. ${ }^{42}$

Macrophage activation has traditionally been considered a dichotomy, where classically activated M1 macrophages are proinflammatory as a result of IFN- $\gamma$ signaling, whereas alternatively activated M2 macrophages are anti-inflammatory after IL-4 and IL-13 exposure. The same general paradigm has been described in microglia, where M1 versus M2 responses have been reported to influence disease outcome. ${ }^{43}$ However, recent reports have revealed more significant plasticity in macrophage profiles than was previously appreciated. ${ }^{44,45}$ Specifically, macrophages treated with either IL- 4 or IFN- $\gamma$ were capable of reversing their phenotype based on the cytokine milieu. ${ }^{44}$ In addition, it has been suggested that macrophage activation depends on the first signal that is encountered, and that classically activated M1 macrophages are more capable of switching to an anti-inflammatory phenotype. ${ }^{45}$ Therefore, it is possible that simultaneous arginase- 1 and iNOS expression occurs during brain abscess development because macrophages/microglia are adjusting their phenotypes to function in both pro- and anti-inflammatory manners, to simultaneously clear infection and promote wound healing by fibrosis, respectively. Additionally, the region-dependent localization of each cell type may govern specificity based on the local cytokine milieu and presence of bacteria. Indeed, immunofluorescence staining revealed that iNOS expression was more diffuse than arginase- 1 and the former extended near the necrotic zone where bacteria are present. This region is expected to be more proinflammatory based on the active synthesis of mediators required for bacterial clearance. In contrast, arginase-1 expression was more restricted to the outermost domains of the abscess, in direct contact with surrounding noninfected parenchyma and where active fibrosis occurs to sequester the lesion. Moreover, ongoing in vitro studies in our laboratory have indicated that if macrophages or microglia are programmed toward a M2 alternative activation state via IL-4 treatment, they remain capable of expressing low levels of iNOS in response to $S$. aureus stimulation, in addition to the large amount of arginase- 1 that is induced (A. Aldrich and T. Kielian, unpublished observations). This finding is in agreement with previous studies demonstrating macrophage plasticity in response to changing stimuli. ${ }^{44,45}$ Currently, there is no method to specifically ablate M2 macrophages/microglia to demonstrate their role in orchestrating brain abscess encapsulation without potentially affecting M1 subsets, since it is 
likely that cells express some degree of M1/M2 marker overlap. Indeed, we and others have found that macrophages and microglia often coexpress M1- and M2-associated molecules (Figure 6), ${ }^{44}$ making the depletion of one subset difficult at best. In addition, evidence indicates that macrophage/microglial polarization is a plastic event influenced by the local cytokine milieu ${ }^{45}$; therefore, it would be difficult to predict whether depletion strategies would target the correct population in vivo. It is possible to ablate M2-associated genes such as arginase in mononuclear cells using LysM-Cre mice. ${ }^{46}$ However, this would only ablate arginase- 1 activity and not the presence of M2 cells per se. Another avenue is to assess the general impact of macrophages/microglia on abscess capsule formation by depleting populations using Macrophage Fas-Induced Apoptosis (MAFIA) or CD11b-diptheria toxin (DT) transgenic mice ${ }^{47,48}$ However, it is important to note that microglia may not be effectively ablated using these approaches, and might prove sufficient to drive the fibrosis program. Another approach involves the adoptive transfer of M2-skewed macrophages to determine effects on abscess fibrotic wall formation. This approach has been used by Weber et $\mathrm{al}^{4}{ }^{49}$ where adoptive transfer of M2 monocytes induced by glatiramer acetate were capable of attenuating disease severity in a mouse experimental autoimmune encephalomyelitis model concomitant with enhanced Th2 differentiation and $\mathrm{T}_{\text {reg }}$ expansion. Our initial attempts with $\mathrm{M} 2$ macrophage transfer did not reveal trafficking of $\mathrm{GFP}^{+}$ M2 macrophages to the brain and will require additional evaluation in future studies. Nonetheless, it remains possible that the plasticity of M1-M2 macrophage populations due to influences of the local cytokine environment will make this analysis difficult. Therefore, because of the limitations in currently available model systems, the functional impact of M2 macrophages/microglia waits testing in future studies.

In summary, this study demonstrates that bone marrow-derived cells can enter the infected CNS and transform into $\alpha-\mathrm{SMA}^{+}$myofibroblasts that associate with the fibrotic abscess capsule. At least a portion of these cells are derived from fibrocyte-like cells, a bone marrow precursor that has been shown to transition into fibroblasts/ myofibroblasts in other models of fibrosis. ${ }^{6}$ In addition, alternatively activated M2 macrophages and microglia were found to accumulate during the course of brain abscess development, where they primarily localized to the outermost regions coincident with fibrotic wall formation. Finally, the expression of genes associated with alternative M2 macrophage/microglial activation and fibrosis all peaked during the time when wall formation is known to commence. Collectively, these studies have laid the foundation for future work to identify the potential mechanisms by which these events occur and the functional importance of bone marrow-derived cells in CNS fibrosis.

\section{Acknowledgments}

We thank Amanda Angle for excellent technical assistance, Dr. Costi Sifri for the USA300 isolate, and Dr.
Charles Kuszynski, Megan Michalak, and Victoria Smith in the UNMC Cell Analysis Facility for assistance with FACS analysis. We thank Kari Nelson for editorial assistance during the preparation of this manuscript.

\section{References}

1. Townsend GC, Scheld WM: Infections of the central nervous system. Adv Intern Med 1998, 43:403-447

2. Kielian T, Phulwani NK, Esen N, Syed MM, Haney AC, McCastlain K, Johnson J: MyD88-dependent signals are essential for the host immune response in experimental brain abscess. J Immunol 2007, 178:4528-4537

3. Kielian T: Immunopathogenesis of brain abscess. J Neuroinflammation 2004, 1:16

4. Flaris NA, Hickey WF: Development and characterization of an experimental model of brain abscess in the rat. Am J Pathol 1992 141:1299-1307

5. Hinz B: The myofibroblast: paradigm for a mechanically active cell. J Biomech 2010, 43: 146-155

6. Bellini A, Mattoli S: The role of the fibrocyte, a bone marrow-derived mesenchymal progenitor, in reactive and reparative fibroses. Lab Invest 2007, 87:858-870

7. Kielian T, Syed MM, Liu S, Phulwani NK, Phillips N, Wagoner G, Drew $P D$, Esen $N$ : The synthetic peroxisome proliferator-activated receptorgamma agonist ciglitazone attenuates neuroinflammation and accelerates encapsulation in bacterial brain abscesses. J Immunol 2008, 180:5004-5016

8. Pilling D, Fan T, Huang D, Kaul B, Gomer RH: Identification of markers that distinguish monocyte-derived fibrocytes from monocytes, macrophages, and fibroblasts. PLoS One 2009, 4:e7475

9. Chesney J, Metz C, Stavitsky AB, Bacher M, Bucala R: Regulated production of type I collagen and inflammatory cytokines by peripheral blood fibrocytes. J Immunol 1998, 160:419-425

10. Valledor AF, Comalada M, Santamaria-Babi LF, Lloberas J, Celada A: Macrophage proinflammatory activation and deactivation: a question of balance. Adv Immunol 2010, 108:1-20

11. Garg S, Nichols JR, Esen N, Liu S, Phulwani NK, Syed MM, Wood WH, Zhang $Y$, Becker KG, Aldrich A, Kielian T: MyD88 expression by CNS-resident cells is pivotal for eliciting protective immunity in brain abscesses. ASN Neuro 2009, 1:e00007

12. Colton CA: Heterogeneity of microglial activation in the innate immune response in the brain. J Neuroimmune Pharmacol 2009, 4:399418

13. Gordon S, Martinez FO: Alternative activation of macrophages: mechanism and functions. Immunity 2010, 32:593-604

14. Meneghin A, Hogaboam CM: Infectious disease, the innate immune response, and fibrosis. J Clin Invest 2007, 117:530-538

15. Sifri CD, Park J, Helm GA, Stemper ME, Shukla SK: Fatal brain abscess due to community-associated methicillin-resistant Staphylococcus aureus strain USA300. Clin Infect Dis 2007, 45:e113-e117

16. Simard AR, Rivest $S$ : Bone marrow stem cells have the ability to populate the entire central nervous system into fully differentiated parenchymal microglia. FASEB J 2004, 18:998-1000

17. Ford AL, Goodsall AL, Hickey WF, Sedgwick JD: Normal adult ramified microglia separated from other central nervous system macrophages by flow cytometric sorting. Phenotypic differences defined and direct ex vivo antigen presentation to myelin basic protein-reactive CD4+ T cells compared. J Immunol 1995, 154:4309-4321

18. Gomperts BN, Strieter RM: Fibrocytes in lung disease. J Leukoc Biol 2007, 82: 449-456

19. Kielian T, Haney A, Mayes PM, Garg S, Esen N: Toll-like receptor 2 modulates the proinflammatory milieu in Staphylococcus aureus-induced brain abscess. Infect Immun 2005, 73:7428-7435

20. Kisseleva T, Uchinami H, Feirt N, Quintana-Bustamante O, Segovia JC, Schwabe RF, Brenner DA: Bone marrow-derived fibrocytes participate in pathogenesis of liver fibrosis. J Hepatol 2006, 45:429-438

21. Phillips RJ, Burdick MD, Hong K, Lutz MA, Murray LA, Xue YY, Belperio JA, Keane MP, Strieter RM: Circulating fibrocytes traffic to the lungs in response to CXCL12 and mediate fibrosis. J Clin Invest 2004, 114:438-446 
22. Sofroniew MV: Molecular dissection of reactive astrogliosis and glial scar formation. Trends Neurosci 2009, 32:638-647

23. Gordon S: Alternative activation of macrophages. Nat Rev Immunol 2003, 3:23-35

24. Lee S, Huen S, Nishio H, Nishio S, Lee HK, Choi BS, Ruhrberg C, Cantley LG: Distinct macrophage phenotypes contribute to kidney injury and repair. J Am Soc Nephrol 2011, 22:317-326

25. Liu SL, Kielian T: MyD88 is pivotal for immune recognition of Citrobacter koseri and astrocyte activation during CNS infection. J Neuroinflamm 2011, 8:35

26. Wynn TA, Barron L: Macrophages: master regulators of inflammation and fibrosis. Semin Liver Dis 2010, 30:245-257

27. Wynn TA: Fibrotic disease and the $\mathrm{T}(\mathrm{H}) 1 / \mathrm{T}(\mathrm{H}) 2$ paradigm. Nat Rev Immunol 2004, 4:583-594

28. Huang LE, Gu J, Schau M, Bunn HF: Regulation of hypoxia-inducible factor 1alpha is mediated by an O2-dependent degradation domain via the ubiquitin-proteasome pathway. Proc Natl Acad Sci U S A 1998, 95:7987-7992

29. He ZJ, Huang ZT, Chen XT, Zou ZJ: Effects of matrix metalloproteinase 9 inhibition on the blood brain barrier and inflammation in rats following cardiopulmonary resuscitation. Chin Med J (Engl) 2009, 122: $2346-2351$

30. Abe R, Donnelly SC, Peng T, Bucala R, Metz CN: Peripheral blood fibrocytes: differentiation pathway and migration to wound sites. J Immunol 2001, 166:7556-7562

31. Mori L, Bellini A, Stacey MA, Schmidt M, Mattoli S: Fibrocytes contribute to the myofibroblast population in wounded skin and originate from the bone marrow. Exp Cell Res 2005, 304:81-90

32. Mooney JE, Rolfe BE, Osborne GW, Sester DP, van Rooijen N, Campbell GR, Hume DA, Campbell JH: Cellular plasticity of inflammatory myeloid cells in the peritoneal foreign body response. Am J Pathol 2010, 176:369-380

33. Frisen J, Goritz C, Dias DO, Tomilin N, Barbacid M, Shupliakov O: A pericyte origin of spinal cord scar tissue. Science 2011, 333:238-242

34. Duffield JS, Schrimpf C: Mechanisms of fibrosis: the role of the pericyte. Curr Opin Nephrol Hy 2011, 20:297-305

35. Stenzel W, Soltek S, Schluter D, Deckert M: The intermediate filament GFAP is important for the control of experimental murine Staphylococcus aureus-induced brain abscess and Toxoplasma encephalitis. J Neuropathol Exp Neurol 2004, 63:631-640

36. Hirano S, Yonezawa T, Hasegawa H, Hattori S, Greenhill NS, Davis PF, Sage EH, Ninomiya Y: Astrocytes express type VIII collagen during the repair process of brain cold injury. Biochem Biophys Res Commun 2004, 317:437-443

37. Saito A, Okazaki H, Sugawara I, Yamamoto K, Takizawa H: Potential action of IL-4 and IL-13 as fibrogenic factors on lung fibroblasts in vitro. Int Arch Allergy Immunol 2003, 132:168-176
38. Kawashima R, Kawamura YI, Kato R, Mizutani N, Toyama-Sorimachi N, Dohi T: IL-13 receptor alpha2 promotes epithelial cell regeneration from radiation-induced small intestinal injury in mice. Gastroenterology 2006, 131:130-141

39. Lee CG, Homer RJ, Zhu Z, Lanone S, Wang X, Koteliansky V, Shipley JM, Gotwals P, Noble P, Chen Q, Senior RM, Elias JA: Interleukin-13 induces tissue fibrosis by selectively stimulating and activating transforming growth factor beta(1). J Exp Med 2001, 194:809-821

40. Song E, Ouyang N, Horbelt M, Antus B, Wang M, Exton MS: Influence of alternatively and classically activated macrophages on fibrogenic activities of human fibroblasts. Cell Immunol 2000, 204: 19-28

41. Hesse M, Modolell M, La Flamme AC, Schito M, Fuentes JM, Cheever AW, Pearce EJ, Wynn TA: Differential regulation of nitric oxide synthase- 2 and arginase- 1 by type 1/type 2 cytokines in vivo: granulomatous pathology is shaped by the pattern of $\mathrm{L}$-arginine metabolism. $\mathrm{J}$ Immunol 2001, 167:6533-6544

42. Raes G, De Baetselier P, Noel W, Beschin A, Brombacher F, Hassanzadeh Gh G: Differential expression of FIZZ1 and Ym1 in alternatively versus classically activated macrophages. J Leukoc Biol 2002, 71:597-602

43. Kigerl KA, Gensel JC, Ankeny DP, Alexander JK, Donnelly DJ, Popovich PG: Identification of two distinct macrophage subsets with divergent effects causing either neurotoxicity or regeneration in the injured mouse spinal cord. J Neurosci2009, 29:13435-13444

44. Stout RD, Suttles J: Functional plasticity of macrophages: reversible adaptation to changing microenvironments. J Leukoc Biol 2004, 76: 509-513

45. Porcheray F, Viaud S, Rimaniol AC, Leone C, Samah B, DereuddreBosquet N, Dormont D, Gras G: Macrophage activation switching: an asset for the resolution of inflammation. Clin Exp Immunol 2005 142:481-489

46. Pesce JT, Ramalingam TR, Mentink-Kane MM, Wilson MS, El Kasmi KC, Smith AM, Thompson RW, Cheever AW, Murray PJ, Wynn TA: Arginase-1-expressing macrophages suppress Th2 cytokine-driven inflammation and fibrosis. Plos Pathog 2009, 5:e1000371

47. Cohen DA, Burnett SH, Kershen EJ, Zhang JY, Zeng L, Straley SC, Kaplan AM: Conditional macrophage ablation in transgenic mice expressing a Fas-based suicide gene. J Leukocyte Biol 2004, 75: $612-623$

48. Saito M, Iwawaki T, Taya C, Yonekawa H, Noda M, Inui Y, Mekada E, Kimata $Y$, Tsuru A, Kohno K: Diphtheria toxin receptor-mediated conditional and targeted cell ablation in transgenic mice. Nature Biotechnol 2001, 19:746-750

49. Weber MS, Prod'homme T, Youssef S, Dunn SE, Rundle CD, Lee L, Patarroyo JC, Stuve O, Sobel RA, Steinman L, Zamvil SS: Type II monocytes modulate $T$ cell-mediated central nervous system autoimmune disease. Nat Med 2007, 13:935-943 